\title{
Influencia de un programa de intervención en las conductas de los entrenadores de baloncesto en categorías de formación
}

\author{
Influence of an intervention program on the behaviors \\ of basketball coaches in youth categories
}

\section{Influência de um programa de intervençáo em comportamentos treinadores de basquete categorias de formação}

\author{
Benjamín Longarela Pérez y Antonio Montero Seoane \\ Facultad de Ciencias del Deporte y la Actividad Física. Universidade da Coruña.
}

\begin{abstract}
Resumen: La presente investigación analiza las conductas de los entrenadores de baloncesto después de lanzamiento a canasta de los jugadores de su equipo y del rival en situaciones de competición. Intervinieron en el estudio seis entrenadores varones de equipos que competían en la liga autonómica de Galicia (España) y en la liga provincial de Lugo (Galicia, España) en las temporadas 2010-2011 y 2011-2012. De los equipos participantes, 5 eran de género masculino y 1 femenino, con una edad de los deportistas comprendida entre 11 y 17 ańos. El instrumento utilizado para el registro de las conductas de los entrenadores procede de la adaptación espańola del Coach Behavioral Assessment System y del Sistema de Observación de las Conductas del Entrenador. Se comparan los resultados obtenidos entre dos fases, teniendo en cuenta que entre ellas se realiza una fase de intervención con cada uno de los entrenadores participantes. De los resultados obtenidos, se puede afirmar que se producen cambios en los comportamientos de los entrenadores tras la fase de intervención.

Palabras clave: behaviorismo, muestra, variables situacionales.

Abstract: This research examines the behavior of coaches basketball after shot of the players of his team and opponent team in competition. In this study, six coaches participed. It was analyzed 38 official games of the Galician league (Spain) and the provincial league of Lugo (Galicia, Spain) in 2010-2011 and 2011-2012 seasons. Of the teams that participate, five were male and one female, with an age of athletes between 11 and 17 years. The
\end{abstract}

instrument used to record the behavior of coaches comes from the Spanish adaptation of the Coach Behavioral Assessment System and Sistema de Observación de las Conductas del Entrenador. We compared the obtaineds results of the two phases, with an intervention's phase made from the coaches in between the first and the second phase. After the results obtained, we can say that there are changes in the behavior of the coaches after the intervention phase.

Keywords: behaviorism, sample, situational variables.

Resumo: Esta pesquisa analisa o comportamento de treinadores de basquetebol após o arremesso dos jogadores da sua equipe e da adversária em situaçōes de competição. Participaram do estudo seis treinadores do sexo masculino em competiçôes na liga regional da Galícia (Espanha) e no campeonato provincial de Lugo (Galícia, Espanha), nas temporadas 2010-2011 e 2011-2012. Das equipes participantes, cinco eram do sexo masculino e uma do sexo feminino, com atletas entre 11 e 17 anos. $\mathrm{O}$ instrumento utilizado para registrar o comportamento dos treinadores vem da adaptação espanhola do Coach Behavioral Assessment System e do Sistema de Observación de las Conductas del Entrenador. Sáo comparados os resultados entre duas fases, sendo que entre elas foi desenvolvido um programa de intervençáo com cada um dos treinadores. A partir dos resultados é possível afirmar que a intervenção proporcionou mudanças no comportamento dos treinadores. Palavras chave: behaviorismo, amostra, variáveis situacionais.

\section{Introducción}

Los entrenadores deportivos son un grupo ampliamente tratado en el ámbito de la psicología del deporte (Gallimore y Tharp, 2004; Gilbert y Trudel, 2004; Smith y Smoll, 2009; Tharp y Gallimore, 1976,) y, dentro de estos trabajos, una línea de investigación analiza sus intervenciones en los entrenamientos y en los partidos, empleando para ello, diversos instrumentos (Smith, Smoll y Curtis, 1979; Miller, 1992; Smith, 1993; More, Mcgarry, Partridge y Franks, 1996; Smith y Smoll, 1990 y 1997).

Dirección para correspondencia [Correspondence address]: Benjamín Longarela Pérez. Ronda del Carmen n $442^{\circ}$ Derecha. La Coruña (España) E-mail: b.longarela@udc.es
El empleo de estos instrumentos se ha orientado a objetivos psicológicos y pedagógicos, centrándose, prioritariamente, en los deportes de equipo (Rutten, Dekovic, Stams, Schuengel, Hoeksma y Biesta, 2007; González 2010). Estos deportes se caracterizan por la conjunción del esfuerzo y la habilidad de diversos deportistas para alcanzar un objetivo común, mediante la consecución de goles, canastas o acciones similares que determinan el resultado de la competición.

La selección de la competición en baloncesto como ámbito de investigación, obedece a las numerosas posibilidades de interacción entre jugadores, árbitros y entrenadores que proporciona, reglamentaria y funcionalmente, esta moda- 
lidad deportiva. En este sentido, Cruz y Capdevila (1997) afirman que las respuestas de los entrenadores en situaciones de competición están condicionados por unos antecedentes e implican unas consecuencias. De este modo, sería posible comprender los diferentes comportamientos individuales, utilizando la metodología adecuada para realizar un estudio de campo, como ocurre entre los que adoptan la competición deportiva como escenario de investigación (Smith, Smoll y Hunt, 1977). Conocer las instrucciones del entrenador durante el partido y analizar su comportamiento verbal, posibilitará el establecimiento de determinados modelos de entrenador (Feu, Ibáńez, Graça, y Sampaio, 2007).

Diversos trabajos inciden en la relevancia del lanzamiento en baloncesto como predictor del resultado de los encuentros (Ibáñez, Lozano y Martínez, 2001; Claramount y Balagué, 2010; Wissel, 2010) a lo que se debe añadir la importancia que le atribuyen los propios deportistas (Buñuel, Sánchez, Fuentes-Guerra y Godoy, 2007; Montero, Ezquerro y Saavedra, 2009; Ortega, Palao, Sáinz de Baranda y García, 2009); por ello, se ha considerado de interés conocer las reacciones de los entrenadores tras el lanzamiento a canasta por parte de los jugadores de su equipo y de los componentes del equipo adversario.

El objetivo de este estudio consiste en describir las conductas de los entrenadores tras el lanzamiento a canasta en situaciones de competición y compararlas entre dos fases de grabación, teniendo en cuenta que entre ambas se realiza un programa de intervención.

Este propósito se concretó en los siguientes objetivos específicos:

1. Describir las conductas de los entrenadores en función de las categorías establecidas tras lanzamientos del equipo del entrenador analizado y del equipo contrario.

2. Comparar las conductas de los entrenadores entre una y otra fase de grabación.

\section{Método}

\section{Participantes}

Intervinieron en el presente estudio seis entrenadores varones $(\mathrm{M}=28,08 ; \mathrm{SD}=3,09$ años) de equipos que competían en la liga autonómica de Galicia (España) y en la liga provincial de Lugo (Galicia, España) en las temporadas 2010-2011 y 2011-2012. Todos los entrenadores disponían de la cualificación necesaria para ejercer como entrenadores en la categoría donde competía su equipo (dos con nivel III, el más elevado en España y cuatro con nivel II).

Participaron en este trabajo 6 equipos, 5 masculinos y 1 femenino, con una edad de los deportistas comprendida entre 11 y 17 años. Se ha solicitado y obtenido por escrito, el consentimiento de todos los sujetos participantes, así como la correspondiente aprobación del Comité de Ética de la Universidad de A Coruña (número de salida: CE 05/ 2014), para realizar el estudio. Se registraron un total de 38 partidos oficiales (18 durante la fase 1 y 20 en la fase 2) distribuidos como aparecen en la tabla 1.

Tabla 1. Número de partidos grabados en cada temporada.

\begin{tabular}{lcccc}
\hline Temporadas & \multicolumn{2}{c}{$2010 / 2011$} & \multicolumn{2}{c}{$2011 / 2012$} \\
\hline Fases & F.1 & F.2 & F.1 & F.2 \\
L. Autonómica & 9 & 12 & 3 & 3 \\
L. Provincial & - & - & 6 & 5 \\
Total & \multicolumn{2}{c}{21} & & \multicolumn{2}{c}{17} & \\
\hline
\end{tabular}

\section{Entrevista con el entrenador}

Se realizó una entrevista estructurada con cada entrenador en la semana previa al primer partido, siguiendo las indicaciones de Fontana y Frey (2000) y Weiss (1994), para conocer su opinión sobre tres bloques de contenidos: el deporte en general, otro destinado al baloncesto, y un tercero, referido a su equipo con especial énfasis en el lanzamiento a canasta. La duración aproximada de la entrevista fue de 60 minutos y se realizó en un ambiente formal y distendido.

\section{Instrumento para la observación de las conductas del entrenador}

El instrumento utilizado para el registro de las conductas procede de la adaptación española del CBAS (Smith et al., 1977) elaborado por Cruz (1989) y del SOCE (Sistema de Observación de la Conducta del Entrenador) elaborado por Montero (2004). Para realizar esa adaptación, se analizaron las diferentes opciones de acciones de tiro en baloncesto y las posibles conductas del entrenador, registrando el resultado del lanzamiento, siguiendo a Montero (2004) y a qué jugador se dirigía el entrenador (dirección). Para ello se consideró "acierto" cuando el lanzamiento sumaba o posibilitaba sumar puntos para el equipo del entrenador observado (por ejemplo, el lanzador recibía una falta en su acción de tiro) y, también, cuando fallaban su lanzamiento los jugadores del equipo contrario; en sentido inverso, se consideró "error" cuando anotaban sus lanzamientos los jugadores del equipo contrario y aquellos lanzamientos errados por los jugadores de su equipo.

\section{Conductas del entrenador}

Las conductas registradas se clasificaron en positivas ("refuerzo tras acierto", "ánimo contingente tras error", "ánimo general", "instrucciones técnicas tras acierto y tras error" y "preguntas al jugador tras acierto y tras error"); negativas 
(“castigo" e "instrucción técnica punitiva”); conductas de inhibición ("ignora error" y "no refuerzo tras acierto") y, un último grupo, denominado "otras conductas” que incluía "rebote", "otras conductas tras acierto y tras error" e "instrucción táctica general”.

\section{Instrumentos utilizados para el registro de la conducta}

a) Cámaras de vídeo, cintas de vídeo y sonido

Para la grabación de los partidos se utilizaron dos cámaras de vídeo, de manera que una seguía la acción de juego mientras que la otra se centraba en el entrenador, sincronizadas con un micrófono.

\section{b) Hojas de registro}

Para la codificación y registro de las variables consideradas en el presente estudio, se diseńó una hoja de registro que incluye: tipo de lanzamiento, número del jugador que lanza, conducta del entrenador; momento en que se emite dicha conducta, a quién se dirige el entrenador y el marcador cuando se efectúa el lanzamiento.

\section{Procedimiento estadístico}

Para el tratamiento estadístico se ha utilizado el programa SPSS en su versión 15.0. Para comprobar la relación de los grupos de conductas entre la fase 1 y la fase 2 se han utilizado las pruebas: coeficiente de contingencia y Phi V de Cramer (nivel de significación $p \leq 0,05$ ).

Para comprobar el grado de acuerdo entre los distintos observadores (inter- observadores), así como la concordancia entre ellos mismos (intra-observadores), se utilizó el índice de Kappa de Cohen.

\section{Entrenamiento y fiabilidad de los observadores}

Dos licenciados en Ciencias de la Actividad Física y el Deporte, especializados en baloncesto, completaron las tareas de entrenamiento y observación, hasta alcanzar o superar un nivel de 0,80 de frabilidad. Los resultados obtenidos por cada observador y entre ellos en las distintas fases de grabación del presente estudio se expresan en las tablas 2 y 3 .

Tabla 2. Valores de la concordancia (fiabilidad inter-observador)

\begin{tabular}{lcc}
\hline Kappa de Cohen & \multicolumn{2}{c}{ Valores } \\
\hline Observadores & Fase 1 & Fase 2 \\
$1-2$ & 0,93 & 0,80 \\
\hline
\end{tabular}

Tabla 3. Valores de la concordancia (fiabilidad intra-observador)

\begin{tabular}{lcc}
\hline Kappa de Cohen & \multicolumn{2}{c}{ Valores } \\
\hline Observadores & Fase 1 & Fase 2 \\
$1-1$ & 0,87 & 0,92 \\
$2-2$ & 0,93 & 0,91 \\
\hline
\end{tabular}

Fases de grabación e intervención con el entrenador

El análisis de las conductas de los entrenadores se realizó en las siguientes etapas:

1.- Fase de grabación 1: en ella se procedió a la grabación de 3 partidos de cada entrenador durante la liga regular.

2.- Fase de intervención: tras finalizar la fase 1, se realizó una fase de intervención con una duración aproximada de 60 minutos que constaba de las siguientes partes:

a) Valoración del entrenador acerca de los resultados de los 3 partidos analizados, su comportamiento durante los encuentros y el análisis de la "reactividad" (Anguera, 1983), o interferencia que el observador, puede ejercer en sus conductas.

b) Presentación, con una duración aproximada de 15 minutos, siguiendo las orientaciones propuestas por Smith y Smoll (2009), donde se le explica al entrenador la importancia y los beneficios de crear un ambiente de "reforzamiento positivo" en su equipo. A continuación, se proyecta un vídeo (de $10 \mathrm{minu}$ tos, aproximadamente) con ejemplos en primer lugar, de las conductas que los entrenadores deberían intentar reducir y, en segundo lugar, aquellas que deberían intentar mantener o incrementar, dada la importancia de un estilo positivo de comunicación basado en el apoyo y la ausencia de punición para la promoción de un clima de implicación a la tarea (Götze y Becker, 2002; Cervelló, Escartí y Guzmán, 2007; Torregrosa, Sousa, Viladrich, Villamarín y Cruz, 2008; Trninic, Papic y Trininic, 2009; Sánchez-Oliva, Marcos, Sánchez-Miguel, Alonso y García-Calvo, 2010; Laforge, Sullivan y Bloom, 2012).

c) Presentación y explicación de los resultados de las conductas en los tres primeros partidos analizados. Tras mostrar los datos obtenidos, el entrenador realizaba una valoración de su comportamiento, del procedimiento utilizado para el análisis de las conductas y de los objetivos planteados por el entrenador para su equipo hasta este momento.

3.- Fase de grabación 2: en esta fase se realiza la grabación de otros 3 partidos con el mismo procedimiento que el señalado en la Fase 1.

4.- Intervención final: se presentan los resultados del estudio a cada uno de los entrenadores, analizando las 
diferencias entre los datos de las fases de grabación 1 y 2; resaltando los cambios registrados en su comportamiento. Esta intervención incluye una entrevista para solicitar su valoración del estudio realizado y de la temporada finalizada respecto a los objetivos definidos y los resultados deportivos esperados.

\section{Resultados}

\section{Conductas observadas de cada entrenador}

La media de conductas por partido de cada entrenador participante en el presente estudio es de 441,33 (SD=110,38), más concretamente la media en la fase 1 es de 409 y la media en la fase 2 es de 472 conductas por partido.

A continuación, en la tabla 4, se presentan los valores de las conductas de los entrenadores en cada fase.

Tabla 4. Frecuencias absolutas y relativas de las conductas agrupadas de los entrenadores diferencias por fases.

\begin{tabular}{|c|c|c|c|c|c|}
\hline \multicolumn{2}{|r|}{$\mathrm{N}=5297$} & Positivas & Negativas & De inhibición & Otras \\
\hline \multirow{5}{*}{$\begin{array}{l}\text { F.1 } \\
(n=2462)\end{array}$} & n (\%) & $568(23,1)$ & $199(8,1)$ & $1363(55,4)$ & $332(13,5)$ \\
\hline & Frecuencia por minuto & 0,78 & 0,27 & 1,89 & 0,46 \\
\hline & Frecuencia Esperada & 624.7 & 193.8 & 1290.3 & 353.2 \\
\hline & Residuo & -56.7 & 5.2 & 72.7 & -21.2 \\
\hline & Residuos tipificados & -2.3 & .4 & 2.0 & -1.1 \\
\hline \multirow{5}{*}{$\begin{array}{l}\text { F.2. } \\
(n=2835)\end{array}$} & n (\%) & $776(27,4)$ & $218(7,7)$ & $1413(49,8)$ & $428(15,1)$ \\
\hline & Frecuencia por minuto & 0,97 & 0,26 & 1,76 & 0,53 \\
\hline & Frecuencia esperada & 719.3 & 223.2 & 1485.7 & 406.8 \\
\hline & Residuo & 56.7 & -5.2 & -72.7 & 21.2 \\
\hline & Residuos tipificados & 2.1 & -.3 & -1.9 & 1.1 \\
\hline \multicolumn{2}{|c|}{ Diferencia entre fases (\%) } & $+4,3$ & $-0,4$ & $-5,6$ & $+1,6$ \\
\hline \multicolumn{3}{|c|}{ Coeficiente de contingencia $=0,61$} & \multicolumn{3}{|c|}{ Nivel Significación $=<0,001$} \\
\hline \multicolumn{3}{|c|}{ V de Cramer = 0,61 } & \multicolumn{3}{|c|}{ Nivel Significación $=<0,001$} \\
\hline
\end{tabular}

Como se puede observar en la tabla 4 , mediante el coeficiente de contingencia y la V de Cramer, se puede afirmar que la relación entre las distintas variables es baja y que existen diferencias significativas entre los diferentes grupos de conductas entre la fase 1 y la fase 2 . Los residuos tipificados de la fase 2 muestran que las conductas positivas (2.1) y el grupo otras conductas (1.1) son superiores a las esperadas, mientras que el -1.9 obtenido por las conductas de inhibición muestra que aparecen menos de las esperadas. Como muestran los resultados, las conductas positivas aumentan su frecuencia en mayor medida tras la fase de intervención con el entrenador, mientras que el grupo "otras conductas" lo hace en menor medida; por el contrario, las conductas que muestran una mayor disminución de la fase 1 a la fase 2 , son las de inhibición y las negativas.

En las tablas siguientes se presentan las conductas de los entrenadores tras lanzamientos de su equipo (A) y del equipo contrario (B); diferenciando, además, cuando se producen tras un acierto o un error en el tiro. De esta manera, en la tabla 5, se presentan los datos correspondientes a las conductas registradas tras lanzamientos de su propio equipo (A).
Tabla 5. Frecuencias relativas de las conductas agrupadas en relación a los lanzamientos de su equipo.

\begin{tabular}{lcccc}
\hline \multirow{2}{*}{ Equipo A } & \multicolumn{2}{c}{ FASE 1 } & \multicolumn{2}{c}{ FASE 2 } \\
\cline { 2 - 5 } & Canasta & Fallo & Canasta & Fallo \\
\hline Positivas & 46,1 & 26,7 & 51,6 & 26,7 \\
Negativas & 0 & 9,3 & 0 & 7,3 \\
Inhibición & 36,6 & 51,9 & 30,9 & 54,5 \\
Otras conductas & 17,3 & 12,1 & 17,5 & 11,5 \\
$\mathrm{~N}=2789$ & 560 & 730 & 706 & 793 \\
\hline
\end{tabular}

Como se puede observar en la tabla 5, las conductas más frecuentes son las de inhibición (tras aciertos y errores), en ambas fases de grabación. El segundo grupo de conductas más frecuentes en los entrenadores, son las positivas, que muestran un incremento en la fase 2 tras aciertos de sus jugadores. En lo referente a las conductas negativas, se puede observar que la frecuencia es relativamente baja, mientras que el grupo "otras conductas", presenta valores similares en ambas fases tras aciertos y errores en las acciones de tiro.

A continuación, en la tabla 6 se presentan los valores de 
las conductas registradas tras lanzamientos del equipo adversario.

Tabla 6. Frecuencias relativas de las conductas agrupadas de los entrenadores en relación a los lanzamientos del equipo adversario.

\begin{tabular}{lcccc}
\hline \multirow{2}{*}{ Equipo B } & \multicolumn{2}{c}{ FASE 1 } & \multicolumn{2}{c}{ FASE 2 } \\
\cline { 2 - 5 } & Canasta & Fallo & Canasta & Fallo \\
\hline Positivas & 12,7 & 7,7 & 16,1 & 14,2 \\
Negativas & 26,0 & 0,3 & 26,3 & 0,4 \\
Inhibición & 51,0 & 77,9 & 45,4 & 66,4 \\
Otras conductas & 10,3 & 14,1 & 12,2 & 19,0 \\
$\mathrm{~N}=2508$ & 496 & 673 & 597 & 742 \\
\hline
\end{tabular}

En la tabla 6 se presentan los resultados tras lanzamientos del equipo contrario, donde se puede observar un aumento de las conductas positivas en la fase 2 , duplicándose su valor si se producen tras fallos en los lanzamientos; así como también aumenta el grupo "otras conductas". Por su parte, el grupo de conductas de inhibición se reduce (tras aciertos y fallos) mientras que las negativas mantienen sus valores.

\section{Discusión}

Los resultados del presente estudio muestran que las conductas más frecuentes son las "de inhibición” con un 52,4\%, valores superiores a los de Mowat (2004) y Montero (2004). El segundo grupo de conductas más frecuentes son las "positivas" (25,4\%), que triplican las conductas negativas (7,9\%), tendencia similar a la registrada por Millard (1996) en fútbol escolar y por Walters (2011) en su estudio a entrenadores de tenis, rugby y fútbol. En sentido opuesto, los datos registrados por Calpe-Gómez (2012) en su estudio con entrenadores de balonmano señalan que el porcentaje de conductas negativas es mayor que el de positivas. Estas diferencias en los resultados pueden deberse, entre otros factores, a las distintas características de cada deporte analizado, así como a la repercusión de cada tanto obtenido en las distintas disciplinas deportivas.

En lo que respecta a las conductas tras error en el lanzamiento, el presente estudio muestra que las positivas $(10,7 \%)$ son superiores a las negativas $(7,8 \%)$, misma tendencia que la registrada por Cruz (1989) con un $8,58 \%$ de positivas y un $5,06 \%$ de negativas, y Montero (2004) quien obtuvo un 7,0\% de conductas positivas y un $2,5 \%$ de negativas; orientación también similar aunque con valores claramente inferiores a los datos de Cruz, Bou, Ferrández, Martín, Monrás, Monfort y Ruiz (1987) que alcanzaron un $64,6 \%$ de conductas positivas y un $3 \%$ de negativas. En sentido inverso, los datos del presente estudio difieren de los obtenidos por Calpe-Gómez (2012) en el que las conductas positivas (14,9\%) son inferiores a las negativas (24,6\%). Estas diferencias pudieran deberse a la especialidad deportiva practicada ya que, en balonmano, se puede esperar una intervención directa de los jugadores del equipo contrario (incluido el portero) para evitar el tanto del equipo atacante, mientras que en baloncesto, y sobre todo, en las categorías de edad de los participantes en el presente estudio, una acción defensiva que tapone el lanzamiento es poco probable.

La divergencia entre los valores moderados del estudio de Cruz (1989), Montero (2004), y Calpe-Gómez (2012) y los elevados porcentajes de conductas positivas obtenidos por Cruz et al. (1987), podrían deberse a que, los datos del presente estudio y los de Montero (2004) se refieren únicamente, a las conductas tras lanzamiento a canasta, mientras que Cruz et al. (1987), Cruz (1989) y Calpe-Gómez (2012) analizaron todas las acciones de los jugadores en los distintos deportes analizados.

Los resultados del presente estudio muestran que, tras la fase de intervención, se producen cambios en los comportamientos de los entrenadores entre la fase de grabación 1 y la fase 2; más concretamente, las conductas positivas aumentan su frecuencia y las negativas y de inhibición disminuyen, misma tendencia que la registrada por Cruz (1999), Mora, Cruz y Torregrosa (2009) y Conde, Almagro, Sáenz-López y Castillo (2009). Es decir, tras la fase de intervención, los entrenadores se convierten en más activos y más positivos, lo cual podría deberse al desconocimiento por parte del entrenador de sus comportamientos en los primeros partidos grabados, lo que posibilita que puedan modificarlos tras la presentación de los datos registrados y la explicación de las consecuencias que tiene un clima positivo para sus jugadores.

Comparando los resultados del presente trabajo (aumento del grupo de conductas positivas tras la fase de intervención) con los obtenidos en estudios similares en otros deportes empleando la misma herramienta de observación (CBAS) y realizando una intervención para modificar la conducta de los entrenadores; se puede observar una similitud con nuestros datos en los cambios registrados tras dichas intervenciones; de esta manera De Marco, Mancini y West (1997) muestran, en su estudio en beisbol, un aumento de un 39\% en las conductas positivas entre fases; de la misma manera que Cruz, Torregrosa, Sousa, Mora y Viladrich (2010) tras el análisis de 9 entrenadores ( 4 de fútbol y 5 de baloncesto) muestran aumentos en las conductas positivas y una disminución de las punitivas. En consecuencia, el número de conductas positivas por minuto aumenta tras la fase de intervención, mientras que las negativas se mantienen, datos que coinciden con los de Sousa, Smith y Cruz (2008) en fútbol escolar, quienes muestran que las conductas positivas se incrementan y las negativas se reducen. Cabe destacar que en los estudios de Sousa et al. (2008) y Cruz et al. (2010), los entrenadores determinaron previamente las conductas que deseaban aumentar o reducir, mientras que en el estudio de De Marco et al. 
(1997) se analizaron todas las conductas de los entrenadores.

El aumento de conductas positivas, registrado en el presente estudio y en los mencionados anteriormente, podría estar influido por la importancia que supone la figura del entrenador en los deportes de equipo, ya que un determinado estilo de comunicación influye directamente en la cohesión del grupo, mientras que en deportes individuales el comportamiento del entrenador afecta únicamente a un deportista. Además, en los estudios de Sousa et al. (2008) y Cruz et al. (2010) al determinar previamente los entrenadores qué conductas que deseaban aumentar y reducir, podría crear una predisposición favorable para incrementar los comportamientos positivos.

En resumen, se puede afirmar que las conductas más frecuentes de los entrenadores participantes son las de inhibición (tras aciertos y errores de ambos equipos), mientras que tras lanzamientos del equipo A son las positivas tras aciertos de sus jugadores y, tras lanzamientos del equipo contrario (B), las más frecuentes después de canasta del equipo contrario son las negativas.

Tras la fase de intervención con el entrenador, se producen cambios en las conductas de los entrenadores aumentando las conductas positivas mientras que disminuyen las de inhibición y negativas; es decir, los entrenadores se convierten en más activos y más positivos tras la intervención realizada.

\section{Aplicaciones prácticas}

El presente estudio, así como todos aquellos dedicados a que los entrenadores sean conscientes de lo que hacen, cómo lo hacen y las consecuencias que eso tiene en la satisfacción y continuidad de los jugadores son fundamentales para la mejora de nuestro deporte.

La información proporcionada a los entrenadores durante la fase de intervención (inicial y final) acerca de sus comportamientos posibilita realizar cambios en sus conductas incluso durante la propia competición, lo cual se convierte en una herramienta fundamental para los entrenadores y sus jugadores.

La necesidad de conocer el propio estilo de comunicación así como la posibilidad de modificarlo, es un aspecto clave para la formación de los entrenadores en la cual a través de cursos de formación de entrenadores o cursos especializados debería de ser un contenido imprescindible.

\section{Referencias}

1. Anguera, Ma T. (1983). Manual de prácticas de observación. México: Trillas.

2. Buñuel, P., Sánchez, A., Fuentes-Guerra, F. y Godoy. (2007). La autopercepción de las jugadoras de baloncesto expertas respecto a sus procesos de formación. Cultura, Ciencia y Deporte, 7 (3), 35-41.

3. Calpe- Gómez, V. (2012). Relación entre factores situacionales derivados de la competición y la conducta verbal de entrenadores de balonmano. Trabajo de fin de Máster, Valencia.

4. Cervelló, E., Escartí, A. y Guzmán, J. F. (2007). Youth sport dropout from the achievement goal theory. Psicothema, 19 (1), 65-71.

5. Claramount, C. y Balagué, N. (2010). Influencia de las instrucciones técnicas en la efectividad del lanzamiento en baloncesto. Apunts. Educación Física y Deportes, 99, 65-71.

6. Conde., Almagro, B.J., Sáenz-López, P. y Castillo, E. (2009). Intervention and evaluation of the motivational climate transmitted by a basketball coach. Revista de Psicología del Deporte, 18, 357-361.

7. Cruz, J. (1989). Proyecto de Investigación: Influencia del entrenador en la motivación de deportistas jóvenes: su evaluación y su cambio. Universidad Autónoma de Barcelona: Barcelona.

8. Cruz, J. (1999). Factores motivacionales en el deporte infantil y asesoramiento psicológico a entrenadores y padres. En. J. Cruz (Ed.), Psicología del Deporte (pp. 245-268). Madrid: Síntesis.

9. Cruz, J. y Capdevila, Ll. (1997). Evaluación en psicología del deporte. En Gualberto Buela- Casal y J. Carlos Sierra (eds.) Manual de evaluación psicológica. Fundamentos, técnicas y aplicaciones, (pp.924-947). Madrid: Siglo XXI.

10. Cruz, J., Bou, A., Ferrández, J., Martín, M., Monrás, J., Monfort, N. y Ruiz, A. (1987). Avaluació conductual de les interaccions entre entrenadors i jugadors de bàsquet escolar. Apunts. Medicina de l'Esport, 24, 89-98.

11. Cruz. J., Torregrosa, M., Sousa, C., Mora, Á. y Viladrich, C. (2010). Efectos conductuales de programas personalizados de asesoramiento a entrenadores en estilo de comunicación y clima motivacional. Revista de Psicología del Deporte, 20 (1), 179-195.

12. De Marco, G., Mancini, V. y West, D. (1997). Reflections on change: a qualitative and quantitative analysis of a baseball coach's behavior. Journal of Sport Behavior, 20 (2), 135-163.

13. Feu, S., Ibáńez, S., Graça, A. y Sampaio, J. (2007). Evaluación psicométrica del cuestionario de orientación de los entrenadores en una muestra de entrenadores espańoles de balonmano.Psicothema, 19 (4), 699-705.

14. Fontana, A. y Frey, J. (2000). The interview. From structured questions to negotiated text. En N. K. Denzin e Y. S. Lincoln (Eds.) Handbook of Qualitative Research (pp.645-672). Thousand Oaks: Sage.

15. Gallimore, R. y Tharp, R. (2004). What a Coach Can Teach a Teacher, 1975-2004: Reflections and Reanalysis of John Wooden's Teaching Practices. The Sport Psychologist, 2 (18), 119-137.

16. Gilbert, W. y Trudel, P. (2004). Role of the coach: how model youth team sport coaches frame their roles. The Sport Psychologist, 18, 21-43.

17. González, Ma (2010). Evaluación psicológica en el deporte: aspectos metodológicos y prácticos. Papeles del Psicólogo, 3 (31), 250-258.

18. Götze, M. y Becker, B. A. (2002)Comunicaçao entre crianças, pais e treinadores na escolinha esportiva de basquetebol em aulas e eventos esportivos - a perspective a partir dos sujeitos. Revista Movimento, 8 (3), 47-62.

19. Ibáńez, S., Lozano, A. y Martínez, B. (2001). Líneas de investigación en el análisis de las acciones de juego en baloncesto. In S.J. Ibáñez \& M.M. Macías (Eds.) Aportaciones al proceso de enseñanza y el entrenamiento del baloncesto (pp.137-147). Copegraf: Cáceres.

20. Laforge, K., Sullivan, P. y Bloom, G. (2012). Coaching behaviors in Canadian youth sport. Athletic Insight, 4 (3), 251-263.

21. Millard, L. (1996). Differences in coaching behaviours of male and female high school soccer coaches. Journal of Sport Behavior, 19 (1), 19-31.

22. Miller, A. (1992). Systematic observation behavior similarities of various youth sport soccer coaches. The Physical educator, 49, 136-143. 
23. Montero, A. (2004). Evaluación de las conductas del entrenador de baloncesto infantil. Tesis doctoral. Universidade da Coruña, A Coruña.

24. Montero, A., Ezquerro, M. y Saavedra, M. (2009). Application of a token economy program and preferences for basketball players. Revista de Psicología del Deporte, 18 (3), 433-437.

25. Mora A., Cruz, J. y Torregrosa, M. (2009. Effects of a training program in comunication styles of basketball coaches. Revista de Psicología del Deporte, 18 (3), 299 - 302.

26. More, K., Mcgarry, T., Partridge, D. y Franks, I. (1996). A computerassisted analysis of verbal coaching behavior in soccer. Journal of Sport Behavior, 19, 319-337.

27. Mowat, J. (2004). Arousal and behaviour of coaches during competition. Tesis doctoral. Faculty of Human Development, Victoria University of Technology.

28. Ortega, E., Palao, J.M., Sáinz de Baranda, P. y García, L.M. (2009). Preferences and levels of satisfaction in technical and tactical actions and in type of offense and defense utilized in competition by youth basketball players. Revista de Psicología del Deporte, 18 (3), 343-348.

29. Rutten, E., Dekovic, M., Stams, J., Schuengel, C., Hoeksma, J. y Biesta, J. (2007). On-and off-field antisocial and prosocial behavior in adolescent soccer players: a multilevel study. Journal of Adolescence, 31, 371-387.

30. Sánchez-Oliva, D., Marcos, F. M., Sánchez-Miguel, P. A., Alonso, D. y García-Calvo, T. (2010). Relación del clima motivacional creado por el entrenador con la motivación auto- determinada y la implicación hacia la práctica deportiva. Revista Internacional de Ciencias del Deporte, 6(VI), 177-195.

31. Smith, R. (1993). Intervenciones psicoeducativas en el deporte. Madrid: Pirámide.

32. Smith, R. y Smoll, F. (1990). Self-Esteem and children's reactions to youth sport coaching behaviors: a field study of self-enhancement processes. Development Psychology, 26 (6), 987-993.
33. Smith, R. y Smoll, F. (1997). Coaching the coaches: youth sports as a scientific and a applied behavioural setting. Current Directions in Psychological Science, 6 (1), 16-21.

34. Smith, R. y Smoll, F. (2009). Claves para ser un entrenador excelente. Barcelona: INDE.

35. Smith, R., Smoll, F. y Curtis, B. (1979). Coach effectiveness training: A cognitive- behavioral approach to enhancing relationship skills in youth sport coaches. Journal of Sport Psychology, 1, 59-75.

36. Smith, R., Smoll, F. y Hunt, E. (1977). A system for the behavioural assessment of athletic coaches. Research Quaterly, 48 (2), 401-407.

37. Sousa, C., Smith, R. y Cruz, J. (2008). An individualized behavioral goal-setting program for coaches. Journal of clinical sport psychology, 2 258-277.

38. Tharp, R. y Gallimore, R. (1976). Basketball's John Wooden: Wath a coach can teach a teacher. Psychology Today, 9 (8), 74-78.

39. Torregrosa, M., Sousa, C., Viladrich, C., Villamarín, F. y Cruz, J. (2008). El clima motivacional y el estilo de comunicación del entrenador como predictores del compromiso en futbolistas jóvenes. Psicothema, 20 (002), 254-259.

40. Trninic, M., Papic, V. y Trninic, V. (2009). Influence of coach's leadership behavior and process of training on performance and competition efficacy in elite sport. Acta Kinesiológica, 3 (1), 18-25.

41. Walters, S. (2011). Whose Game are we playing? A study of the effects of adult involvement on children participating in organised team sports. Doctoral Thesis. Auckland University of Technology.

42. Weiss, R. (1994). Learning from strangers. The art and method of qualitative interview studies. New York: The Free Press.

43. Wissel, H. (2010). Técnicas de tiro. Cap.1. En Giorgio Gandolfi (Dir.). Libro de jugadas de los entrenadores de la NBA (pp. 25-41). Madrid: Tutor. 